\title{
Análisis de sensibilidad en el cálculo de emisiones contaminantes del tráfico de mercancías en ejes de transporte
}

\author{
Jorge Ruíz Porro \\ Alumno de Máster Ing. Industrial ETSI Industriales, Universidad Politécnica de Madrid \\ jorge.ruiz.porro@gmail.com \\ José Mira McWilliams \\ Profesor Titular de Universidad, ETSI Industriales, Investigador INSIA, UPM \\ josemanuel.mira@upm.es \\ Blanca Arenas Ramírez \\ Profesora Contratada Doctora, ETSI Industriales, Directora de la Unidad de Estudios de \\ Transporte e Impacto Medioambiental de INSIA - UPM - España \\ blanca.arenas@upm.es \\ José M. López Martínez \\ Profesor Titular de Universidad, ETSI Industriales, Director INSIA, UPM - España \\ josemaria.lopez@upm.es \\ Francisco Aparicio Izquierdo \\ Profesor Emérito de la UPM, Presidente de INSIA UPM \\ francisco.aparicio@upm.es
}

\section{RESUMEN}

La competitividad en el transporte de mercancías español y mundial, junto con la preocupación creciente por la reducción de emisiones, ha provocado que se preste una gran atención a la mejora de su eficiencia, tanto desde el punto de vista logístico como el medioambiental. Por ello, el trasvase modal tiene una relevancia creciente en las administraciones y en investigación. En este trabajo se desarrolla un análisis de sensibilidad sobre un código de ordenador de simulación estocástica, que estima la distribución de probabilidad de las emisiones que se dejan de producir en la carretera como consecuencia del trasvase modal de mercancías al ferrocarril. El modelado estadístico proporciona el valor añadido esencial de la cuantificación de las incertidumbres y comprende varias etapas que posteriormente se acoplan mediante el método de Monte Carlo, implementado en un código de simulación escrito en lenguaje abierto $\mathrm{R}$. El análisis de sensibilidad se realiza a través de un experimento computacional cuyos resultados se analizan mediante la técnica ANOVA, para estudiar el efecto, sobre las emisiones, de cambios en las distribuciones de probabilidad de las variables que determina las etapas del modelo, tanto de forma individual (efectos principales) como conjunta (interacciones). La metodología se aplica como ilustración al eje Madrid-Guipúzcoa para la estimación de consumos y de las emisiones de $\mathrm{CO}, \mathrm{NOx}, \mathrm{HC}$ y de material particulado (PM).

\section{INTRODUCCIÓN}

El objetivo de este trabajo es el análisis de sensibilidad para un simulador estocástico 
desarrollado en Arenas et al. (2014), que estima la distribución de probabilidad de las emisiones que se dejan de producir como consecuencia del trasvase modal de mercancías de la carretea al ferrocarril. Se aplica la metodología al eje Madrid-Guipúzcoa (MG).

El trasvase modal es un aspecto importante en Ingeniería del Transporte, Den Boer et al. (2011), por su efecto sobre tanto las emisiones como la accidentalidad, dada la naturaleza estocástica de los factores que afectan al proceso, el enfoque debe ser estadístico, con cuantificación de las incertidumbres.

La estimación de las emisiones resultantes del transporte de mercancías ha sido objeto de mucha investigación, incluyendo la cuantificación de las incertidumbres. Kouridis et al. (2010), y Kiostsoukis et al. (2004) tratan las incertidumbres en torno a los factores de emisión. Capros et al. (2013) realizan un análisis de escenarios con horizonte 2020, El trabajo de Borestein et al. (2014) aplica teoría económica y análisis de series temporales para predecir niveles de emisiones de gases de efecto invernadero en California. Lloyd y Ries (2007) es una revisión de la metodología para incorporar y propagar incertidumbres al análisis de ciclo de vida. Facanha and Horvath (2006) comparan emisiones de diferentes modos de transporte para distintos contaminantes en un contexto de análisis de ciclo de vida $\mathrm{y}$ analizan las incertidumbres.

Una referencia muy clara y con ejemplos interesantes sobre análisis de sensibilidad es Saltelli et al. (2008). Oackley et al. (2001) aplican metodologías muy sofisticadas de campos aleatorios bajo el enfoque bayesiano para estimar la descomposición ANOVA de códigos de ordenador complejos.

En Arenas et al. (2014) se aplicó un enfoque integrado a través de incorporar todas las etapas y las incertidumbres asociadas y a continuación se acoplan, a través de la llamada propagación de incertidumbres que se realiza por simulación de Monte Carlo , implementada en un código escrito en el lenguaje abierto $\mathrm{R}$. El código es una herramienta muy flexible que permite introducir, con muy bajo coste, cambios en los escenarios de transporte con el propósito de evaluar su efecto sobre las emisiones que se dejan de producir en la carretera como consecuencia del trasvase modal. Los efectos principales (individuales) y conjuntos (interacciones) de los cambios sobre las emisiones se analizarán a través de un análisis de sensibilidad tipo ANOVA. La metodología se aplicará a la estimación de consumos y de las emisiones de gases contaminantes (CO, HC, NOx) y material particulado (PM).

El resto de este documento está estructurado como sigue. En la sección 2 se revisan brevemente la estructura y las características probabilistas del simulador, tal y como se desarrollaban en Arenas et al. (2014). En la sección 3 se desarrolla el análisis de sensibilidad $\mathrm{y}$ en la 4 se exponen las conclusiones.

\section{ESTRUCTURA Y CARACTERÍSTICAS DEL SIMULADOR}

Se explica a continuación la estructura y etapas del simulador del análisis de sensibilidad, cuyos cálculos se realizarán por Monte Carlo y se aplicará al eje Madrid-Guipúzcoa (MG).

\subsection{Estructura del simulador}

La secuencia de etapas viene determinada por la siguiente expresión: 


$$
E_{i}=T \times P_{M G} \times p_{T} \times E F_{i}
$$

donde

$T$ : son las t-km anuales en toda España

$P_{M G}$ es la proporción de t-km para el eje MG

$p_{T}$ es la proporción global de mercancías trasvasables de carretera a ferrocarril, que se obtiene a partir de la expresión

$$
p_{T}=\sum_{i=1}^{16} a_{i} p_{i}
$$

donde $p_{i}$ y $a_{i}$ son, respectivamente, las proporciones de mercancías trasvasables para el tipo y el peso relativo de la de mercancía i con respecto al total que se transporta en el eje. El sumatorio se extiende a los 16 tipos de mercancías consideradas en esta aplicación.

$E F_{i}$ es el factor de emisión del contaminante i, y

$E_{i}$ son las emisiones del contaminante i eliminadas de la carretera por el trasvase.

Si se da carácter aleatorio a los factores $T, P_{M G}, p_{T}, E F_{i}$, automáticamente la respuesta $E_{i}$ del primer miembro pasa a ser también aleatoria. Se deben entonces acoplar y propagar las incertidumbres de $T, P_{M G}, p_{T}, E F_{i}$ para poder estimar las distribución de $E_{i}$.

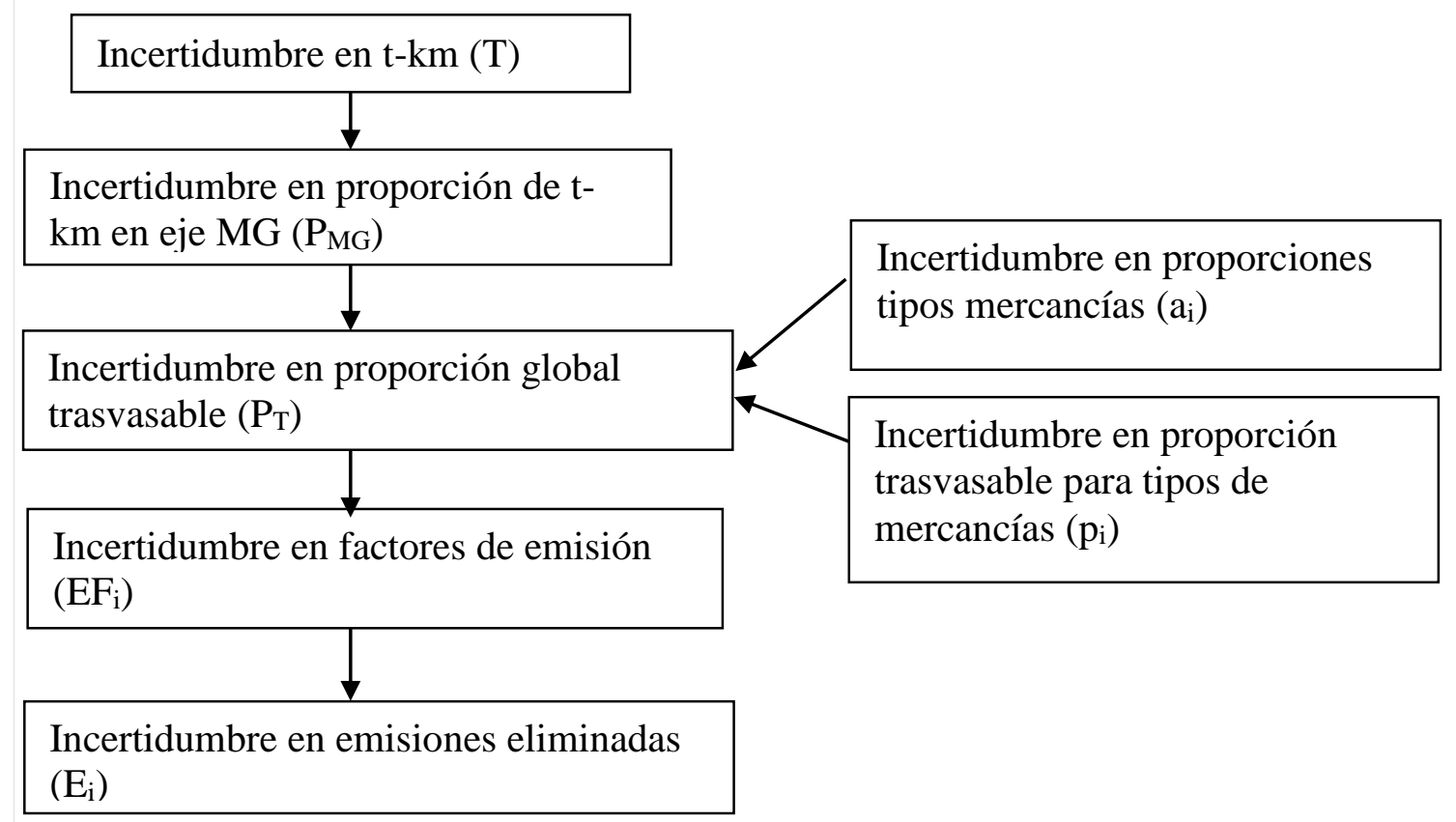

\section{Figura 1: secuencia de cálculos del simulador}




\subsection{Distribuciones de probabilidad para las variables aleatorias del simulador}

La figura 1 presenta esquemáticamente la secuencia de cálculos estocásticos.

$T$ : para el modelado de T se postula, de acuerdo con Witzke et al. (2013), que la tasa de crecimiento anual de las t-km 2020 es constante a lo largo de los años, pero es una realización de una distribución uniforme entre $9,9 \%$ y $1,3 \%$.

$P_{M G}$ sigue una distribución uniforme en un intervalo del $90 \%$ al $110 \%$ en torno a la estimación puntual.

Para los términos $a_{i}$ y $p_{i}$ de proporción total trasvasada $\mathrm{P}_{\mathrm{T}}$ se suponen también distribuciones uniformes.

Los factores de emisión $\mathrm{EF}_{\mathrm{i}}$ son en general distintos para cada contaminante, y se obtienen a partir de expresiones como por ejemplo

$$
\mathrm{EF}=\left(1 /\left(\mathrm{a}+\left(\mathrm{b}^{*}\left(\mathrm{v}^{\wedge} \mathrm{c}\right)\right)\right)\right)
$$

Para cuantificar la incertidumbre, se construyen intervalos del $90 \%$ al $110 \%$ en torno a los valores nominales de las constantes a, b, c y la velocidad v. La incertidumbre de estas 4 variables aleatorias se propaga a EF a través de la expresión [3], determinando así la distribución de probabilidad de EF.

\subsection{Acoplamiento de las incertidumbres}

Las incertidumbres asociadas a la expresión [1] se acoplan y propagan por Monte Carlo, de forma que se generan muestras de $T, P_{M G}, p_{T}, E F_{i}$ y a partir de ellas, muestras de $E_{i}$, de forma que se pueden estimar los parámetros de su distribución.

En la figura 2 y en la tabla 1 se presentan, a modo de ilustración, el histograma y la estadística descriptiva para las emisiones de $\mathrm{CO}$ eliminadas de la carretera como consecuencia del trasvase.

\begin{tabular}{|l|l|l|l|l|l|}
\hline Perc. 2,5\% & $\begin{array}{l}\text { Primer } \\
\text { cuartil Q11 }\end{array}$ & Mediana & Media & $\begin{array}{l}\text { Tercer } \\
\text { cuartil }\end{array}$ & $\begin{array}{l}\text { Perc. } \\
97,5 \% .\end{array}$ \\
\hline $0,0037 \times 10+9$ & $0,0044 \times 10+9$ & $0,005 \times 10+9$ & $0,038 \times 10+9$ & $0,0143 \times 10+9$ & $0,337 \times 10+9$ \\
\hline
\end{tabular}

\section{Tabla 1: Estadística descriptiva para las emisiones de CO (g)}

Se observa que el histograma presenta una gran asimetría, con la cola a la derecha, siguiendo el comportamiento (no descrito en esta ponencia por falta de espacio) de la distribución del factor de emisión correspondiente. En la tabla de estadística descriptiva de la muestra de Monte Carlo se observa que la media y la mediana son muy diferentes, de acuerdo con la asimetría del histograma. 


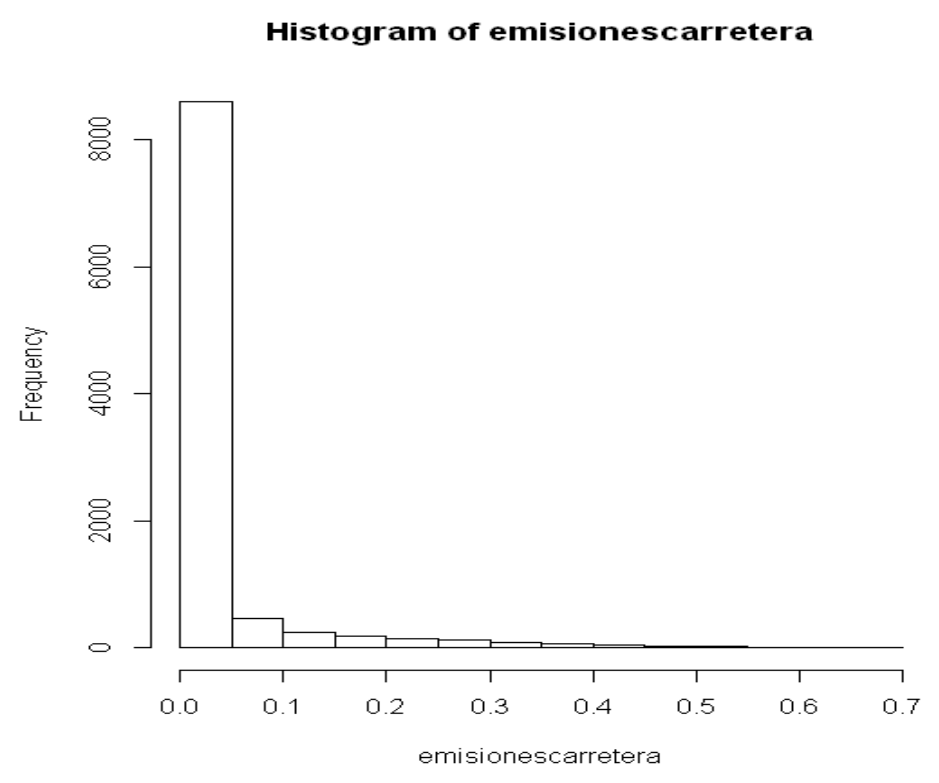

Figura 2: Histograma para emisiones CO

\section{ANÁLISIS DE SENSIBILIDAD A TRAVÉS DE ESCENARIOS}

El análisis de sensibilidad consiste en estudiar, para una relación entrada-salida $Y=Y\left(X_{1}, \ldots, X_{K}\right)$, cómo la variable de salida $(Y)$ se ve afectada por pequeños cambios en las variables de entrada $\left(X_{1}, \ldots, X_{K}\right)$.

Los análisis de sensibilidad admiten clasificaciones según dos criterios. En primer lugar, según se refieran al efecto sobre la respuesta de un cambio local en $\left(X_{1}, \ldots, X_{K}\right)$, es decir en un pequeño intervalo en torno a un punto concreto (análisis de sensibilidad locales) o sean un promedio para todo el rango de variación de las variables de entrada (análisis de sensibilidad globales). En segundo lugar, según se refieran al efecto marginal de una variable o al efecto conjunto de varias variables (interacciones). En este trabajo se aplican técnicas ANOVA para realizar un análisis de sensibilidad global, estimando tanto efectos principales como interacciones.

\subsection{Técnicas basadas en el análisis de la varianza (ANOVA)}

Para un diseño de experimentos ortogonal, la descomposición ANOVA de la suma total de cuadrados en las contribuciones de los efectos principales y las interacciones se puede escribir de forma compacta como sigue:

$$
\begin{gathered}
V T=V E(\alpha)+\cdots+V E(\delta)+V E(\alpha \beta)+\cdots+ \\
+V E(\gamma \delta)+V E(\alpha \beta \gamma)+\cdots+V E(\alpha \beta \gamma \delta)+V E(\alpha \beta \gamma \delta \theta)+V N E)
\end{gathered}
$$

donde: 
$\operatorname{VE}(\alpha)$ - suma de cuadrados para el efecto principal del primer factor

$\operatorname{VE}(\alpha \beta)$ - suma de cuadrados para la interacción de orden dos entre los dos primeros factores.

$\operatorname{VE}(\alpha \beta \gamma)$ - suma de cuadrados para la interacción de orden tres entre los tres primeros factores.

$\operatorname{VE}(\alpha \beta \gamma \delta)$ - suma de cuadrados para la interacción de orden cuatro entre los cuatros primeros factores y así sucesivamente

$V N E$ es la suma de cuadrados residual, que en el caso particular de este experimento computacional, es despreciable ya que solo se debe al cambio de la muestra de Monte Carlo, que ha sido de tamaño igual a 10000.

En este trabajo, el diseño del experimento computacional correspondiente a todas las combinaciones de los niveles de los factores se presenta en la tabla 2.

\begin{tabular}{|l|l|}
\hline Factor & Niveles \\
\hline $\begin{array}{l}\text { Promedio crecimiento anual T-km España } \\
\text { (CREC) }\end{array}$ & 1,011 y 1,02 \\
\hline T-km grupo 10 en eje M-G (TM-G) & $161,1 \times 10^{6}$ \\
\hline $\begin{array}{l}\text { Media intervalo trasvasabilidad alta F } \\
\text { (TRASV) }\end{array}$ & 0,45 y 0,55 \\
\hline Tipo de camión (TIPOCAM) & $\begin{array}{l}\text { Euro IV y Euro VI con ponderación } \\
\text { variable }\end{array}$ \\
\hline
\end{tabular}

Tabla 2 - Factores y niveles de análisis de sensibilidad.

Para el factor "tipo de camión", el número de niveles varía con el horizonte, porque se supone que la proporción de vehículos del tipo EURO VI irá aumentando con el tiempo en detrimento de la de EURO IV. La proporción de EURO VI para cada año hasta el horizonte máximo de 2020 se presenta en la tabla siguiente.

\begin{tabular}{|l|l|l|l|l|l|}
\hline 2015 & 2016 & 2017 & 2018 & 2019 & 2020 \\
\hline $20 \%$ & $35 \%$ & $50 \%$ & $65 \%$ & $80 \%$ & $100 \%$ \\
\hline
\end{tabular}

\section{Tabla 3 - Proporción de EURO IV según el año}

El esquema siguiente representa el diseño del experimento computacional que se analizará mediante la técnica ANOVA.

El análisis de sensibilidad que se presenta a continuación consiste en estudiar el efecto de cambios sobre la respuesta $\mathrm{E}$, de algunos o todos las variables que intervienen en [1], respecto del valor de la respuesta que se ha obtenido para los valores iniciales (originales).

Especifiquemos a continuación a cuál de las variables de [1] afecta a cada uno de los factores de la tabla 2.

1) el promedio de crecimiento afecta a $T$ 
2) las t-km son del grupo 10 afectan a $\mathrm{a}_{10}$ en el sumatorio $\sum_{i}^{16} a_{i} p_{i}$

3) la media de la horquilla de trasvasabilidad afecta a $\mathrm{p}_{2}, \mathrm{p}_{7} \mathrm{y} \mathrm{p}_{10}$

4) el tipo de camión afecta al factor de emisión EF.

A continuación, como ejemplo de ilustración, se presenta la tabla ANOVA para el CO.

\begin{tabular}{|l|l|l|}
\hline Término & Suma de C. & Influencia (\%) \\
\hline CRECMER & $1,2 \mathrm{E}-5$ & $1,3 \mathrm{E}-1$ \\
\hline TM-G & $3, \mathrm{E}-6$ & $3,5 \mathrm{E}-2$ \\
\hline TRASV & $2,5 \mathrm{E}-5$ & $2,9 \mathrm{E}-1$ \\
\hline TIPOCAM & $8,5 \mathrm{E}-3$ & 99,1 \\
\hline CRECMERx TM-G & $7,5 \mathrm{E}-9$ & $8,7 \mathrm{E}-4$ \\
\hline CRECMERx TRASV & $9,6 \mathrm{E}-8$ & $1,1 \mathrm{E}-2$ \\
\hline CRECMERx TIPOCAM & $6,8 \mathrm{E}-6$ & $8,0 \mathrm{E}-2$ \\
\hline TM-Gx TRASV & $3,4 \mathrm{E}-6$ & $3,9 \mathrm{E}-2$ \\
\hline TM-Gx TIPOCAM & $2,0 \mathrm{E}-6$ & $2,3 \mathrm{E}-2$ \\
\hline TRASVXTIPOCAM & $1,4 \mathrm{E}-5$ & $1,7 \mathrm{E}-1$ \\
\hline CRECMERx TM-GxTRASV & $6.1 \mathrm{E}-7$ & $7,1 \mathrm{E}-3$ \\
\hline CRECMERx TM-GxTIPOCAM & $7,9 \mathrm{E}-8$ & $9,2 \mathrm{E}-4$ \\
\hline CRECMERx TRASVxTIPOCAM & $1,1 \mathrm{E}-6$ & $1,2 \mathrm{E}-2$ \\
\hline TM-GxTRASVXTIPOCAM & $3,2 \mathrm{E}-6$ & $3,7 \mathrm{E}-2$ \\
\hline $\begin{array}{l}\text { CRECMERx } \\
\text { GxTRASVxTIPOCAM }\end{array}$ & $6,1 \mathrm{E}-5$ & $7,1 \mathrm{E}-3$ \\
\hline TOTAL & & \\
\hline & $8,5 \mathrm{E}-3$ & 1 \\
\hline
\end{tabular}

Tabla 4-Tabla ANOVA para análisis de sensibilidad de CO.

De esta tabla se obtienen las siguientes conclusiones:

- La contribución de los efectos principales es mucho mayor que la de las interacciones.

- El factor más influyente para las emisiones de CO, con una amplia diferencia con respecto al resto, es el del tipo de camión (Euro IV o Euro VI).

- Todos los demás factores (y combinación de los mismos) tienen un porcentaje de influencia mucho menor. De entre ellos, destaca la influencia de la media de la horquilla de trasvasabilidad de los grupos 2, 7 y 10 de mercancías seguido de la influencia del término compuesto por esta media y el tipo de camión considerado. Se encuentra próximo en influencia el factor de promedio de crecimiento anual en $\mathrm{t} \cdot \mathrm{km}$ en España.

Aunque por falta de espacio no se muestran más tablas de resultados, a modo de resumen se puede señalar que para el resto de los contaminantes los resultados del análisis son muy similares; para el consumo, sin embargo, los efectos principales de la trasvasabilidad y de la tasa de crecimiento (64 y $27 \%$ respectivamente) resultan los más importantes.

\section{CONCLUSIONES}


Se ha realizado un análisis de sensibilidad tipo ANOVA de un modelo estocástico para la estimación de los consumos y de las emisiones que se dejan de producir en la carretera como consecuencia del trasvase de mercancías al ferrocarril. El modelo estocástico no solo proporciona estimaciones puntuales sino también intervalos de incertidumbre.

El análisis se ha realizado a partir de los resultados de un experimento computacional con 4 factores. Para los contaminantes el efecto principal de la tecnología (EURO IV o EURO VI) ha resultado con gran margen de diferencia el factor más importante, mientras que para el consumo lo han sido la trasvasabilidad y la tasa de crecimiento de la movilidad de mercancías (t-km).

\section{AGRADECIMIENTOS}

Este trabajo se ha realizado en el marco del Proyecto de investigación MODALTRAM TRA2011-28647-C02-01 "Desarrollo de una metodología integrada de evaluación de los efectos sobre la seguridad y el medio ambiente por el trasvase modal entre la carretera al ferrocarril", del Plan de Investigación 2011-2014, del Ministerio de Economía y competitividad (MINECO). Además, los autores también quieren agradecer la financiación parcial de este trabajo a la Comunidad de Madrid, que mediante el programa SEGVAUTOTRIES-CM (S2013-MIT2713), ha contribuido al desarrollo del mismo.

\section{REFERENCIAS}

ARENAS, B, MIRA, J., ALEGRE, J.A., LÓPEZ, J.M, GONZÁLEZ, C. y APARICIO, F., (2014), Cuantificación de incertidumbres en el trasvase de tráfico de mercancías en ejes de transportes. Congreso de Ingeniería del Transporte, Santander, Junio 2014.

BORESTEIN, S., BUSHNELL, J., WORAK, F., y ZARAGOZA-WATKINS, M., (2014), Expecting the unexpected: Emission Uncertainty and Environmental Market Design. Annual Power Research Conference.

CAPROS, P., DE VITA, A., TASIOS, N., PAPADOPULOS, D., SISKOS., P., APOSTOLAKIS, E., ZAMPARA, M., PARAUSSOS. M., FRAGIAKIADIS, K., KOUVARUTAKIS, N. (2013), EU Energy, Transport, and GHG Emissions Trends to 2050, Reference Scenario 2013.

DEN BOER, E., VAN ESSESN, H., BROUWER, F., PASTORI, E., MOIZO, A. Potential of modal shift to rail transport - Study on the projected effects on GHG emissions and transport volumes (2011). Delft, CE Delft. Transporti e Territorio SRL (TRT).

FACANHA, C., y HORVATH, A., (2006), "Environmental Assesment of Freight transportation in the U.S.", International Journal of LCA, 11(4), 229-239.

KOURIDIS, C., GATZOFILAS, D., LIOSTSOUKIS, I., NITZIACHIRTOS, L., PASTORELLA, C., and DILLARA, P., (2010), Uncertainty Estimates and Guidance for Road transport Emission Calculations, EUR 242296 JRC Scientific Report. 
LLOYD, S., y Ries, R. (2007), Characterizing, propagating and Analyzing Uncertainty in Life.Cycle Assessment, A survey on Quantitative approaches, Journal of Industrial Ecology, 11(1), pp 161-179.

OACLKEY, J. y O'HAGAN, T., (2004), Probabilistic sensitivity analysis of complex models: a Bayesian approach, Journal of the Royal Statistical Society (B), 66 (3), 751-769. SALTELLI, A., CHAN, A., y SCOTT, E.M., (2008), Sensitivity Analysis. Wiley. WITZKE, A, et al (2013), EU Energy, Transport and GHG Emissions Trends to 2050. Reference scenario 2013 (2013). E3M-Lab of the ICCS-NTUA, Greece. International Institute for Applied Systems Analysis (IIASA). EuroCARE. European Commission. 